\title{
Potentialities of Metallophthalocyanines-Based Gas Sensors for Monocyclic Hydrocarbons Monitoring in Air
}

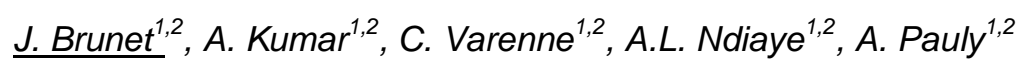 \\ ${ }^{1}$ Clermont Université, Université Blaise Pascal, Institut Pascal, BP 10448, F-63000 Clermont-Ferrand; \\ ${ }^{2}$ CNRS, UMR 6602, Institut Pascal, F-63171 Aubière, France \\ brunet@univ-bpclermont.fr
}

\begin{abstract}
Focused on the development of chemical sensors dedicated to BTX measurements, our recent works have demonstrated the relevance of tetra-tert-butyl metallophthalocyanines (ttb-MPc) as sensitive material for this purpose. Taking into consideration the aromatic character of both the target gases and the sensing material, sorption mechanisms involve $\pi$-stacking interactions without charge transfer between gaseous molecules and phthalocyanine units. Because of the nature of interactions, acoustic transducer and more precisely quartz crystal microbalance were used. Studies achieved on different unsubstituted metallophthalocyanines have revealed the non-significant effects of the central metal atom on the sensor responses and the limited sensitivity towards the target gases. In contrast, the predominant role of electro-donor substituents like tert-butyl groups grafted at the periphery of phthalocyanine macrocycle on sensing characteristics has been clearly established. A 6-time greater sensitivity to BTX was obtained for ttb-MPc as compared to unsubstituted metallophthalocyanines. Response and recovery times at room temperature close to 3 minutes were measured. Sensing performances enhancement observed for ttb-MPc as compared to unsubstituted phthalocyanines are strongly linked to the structure and the morphology of the thin layers as highlighted by SEM characterization. If ttb-MPc-based QCM sensors are insensitive to $\mathrm{H}_{2} \mathrm{~S}, \mathrm{CO}$ and $\mathrm{NO}_{2}$, the discrimination between benzene, toluene and xylene remains a challenge. Ongoing research on this problematic will be finally discussed.
\end{abstract}

Key words: metallophthalocyanine, QCM, acoustic sensors, chemical sensors, volatile organic compounds, air quality control

\section{Motivation}

In 2010, the World Health Organization reported that more than 220000 deaths from lung cancer worldwide resulted from air pollution [1]. In 2012, it was estimated that 7 millions premature deaths per year can be attributed to atmospheric pollution. Based on these public health data, the International Agency for Research on Cancer (IARC) has classified in 2013 outdoor air pollution as carcinogenic to humans [2]. The hazardous consequences of atmospheric pollution on human health are thus so established and the requirement to measure individual exposure of people to pollutants for health prevention is undeniable.

Among pollutants, monocyclic hydrocarbons such as BTEX (Benzene, Toluene, Ethylbenzene and Xylenes) emanating from exhausts, solvents, paints and household products are ubiquitous and strongly hazardous. The International Agency for Research on Cancer (IARC) classifies benzene as carcinogenic to humans (group 1) and ethylbenzene as possibly carcinogenic to humans (group 2B). The international guidelines define personal exposure limits from few ppb to $1 \mathrm{ppm}$ in non-occupational context and in the 10-200 ppm range in occupational context. Because we are daily exposed to these concentration levels in indoor as well as in outdoor environments [3], the continuous monitoring of these pollutants and the evaluation of their effects on human health remain priorities in the context of air quality control.

Despite their good sensing specifications, gas analyzers and passive samplers remain not appropriate for the measurement of individual exposure to pollution. In contrast, the implementation of mobile, low-power and low dimension microsensors in clothes, mobile phones or vehicles could be relevant for such applications. Among sensing performances, high resolution, low threshold of detection, low response time, repeatability, long-term stability and selectivity must be especially satisfied. 


\section{Sensing strategy}

Classified as volatile organic compounds (VOCs), benzene, toluene and xylenes are monocyclic hydrocarbons including only one benzene ring with respectively 0,1 or 2 methyl groups in substitution of peripheral hydrogen atoms. Aromatic molecules like BTX can interact together through non-covalent attractive forces existing between $\pi$-electrons. These weak attraction forces are commonly denoted as $\pi$-stacking interactions [4,5]. Taking into account the aromatic nature of the target gaseous molecules, sensing materials exhibiting a high aromaticity or high $\pi$-electron delocalization were chosen. Due to their four benzene rings and 18 delocalized $\pi$-electrons per molecular units, their easy processability into thin films and their compatibility with several transducing modes, metallophthalocyanines are appropriate molecular material to achieve highly sensitive devices to BTX. Furthermore, $\pi$-stacking involving weak interactions, a complete and easy reversibility at low temperature are expected.

Taking into consideration the chemical properties of the analytes and the interaction mechanisms involved, electronic charge transfer between BTX gases and metallophthalocyanines is unlikely. Preliminary experimental studies on chemoresistive devices have confirmed this postulate. As a consequence, acoustic transducers and more especially quartz crystal microbalance were selected. Different substituted and unsubstituted metallophthalocyanines were layered onto the electrodes of quartz crystals. The influences on sensor response of the central metallic atom and the electroactive properties of peripheral groups grafted in the phthalocyanine macrocycle were especially investigated.

\section{Experimental}

Metallophthalocyanines (MPc with $\mathrm{M}=\mathrm{Fe}, \mathrm{Cu}$, $\mathrm{Co}, \mathrm{Zn})$ free phthalocyanine $\left(\mathrm{H}_{2} \mathrm{Pc}\right)$ and tetratert-butyl metallophthalocyanines (ttb-MPc with $\mathrm{M}=\mathrm{Zn}, \mathrm{Cu}$ ) with purity higher than $90 \%$ were purchased from Sigma Aldrich. $5 \mathrm{MHz}$ AT-cut quartz crystals of diameter $25.4 \mathrm{~mm}$ with $\mathrm{Au} / \mathrm{Cr}$ electrodes were purchased from Maxtek, Inficon. Thin films were realized by thermal evaporation under secondary vacuum at a deposition rate close to $0.2 \mathrm{~nm} / \mathrm{s}$ on quartz crystals maintained at room temperature. No physical or chemical treatment prior and after deposition process has been realized on transducer and thin films. Different thicknesses were studied, in the $25-400 \mathrm{~nm}$ range. The structure and morphology of thin layers were characterized by SEM and FT-IR spectroscopy.

Sensors were characterized under benzene, toluene and xylene vapors by means of homemade and calibrated setup controlled by labview software. The exposure chamber including QCM-based and temperature sensors were made in PTFE. Excited at $5 \mathrm{MHz}$ by a Maxtek PLO-10i oscillator, frequency variations of quartz crystals were recorded by an Agilent 53131A $225 \mathrm{MHz}$ Universal Counter.

The adsorption of gaseous molecules generates a mass variation of the sensitive layer, inducing a modulation of the resonant frequency of the coated quartz crystal according to the Sauerbrey equation (1):

$$
\Delta f=-\frac{C f_{0}^{2} \cdot \Delta m}{A}
$$

with $A$, the area of sensitive layer and $C$, the mass sensitivity constant $\left(=4.816 \times 10^{-10} \mathrm{~m}^{2} \mathrm{~s}\right.$ $\mathrm{g}^{-1}$ ) of quartz crystal. Frequency decreases during gas adsorption and increases during desorption. All experimental results have been obtained at room temperature with residual relative humidity close to $3 \pm 1 \%$. More experimental details are given in reference [6].

\section{Results and discussion}

Fig.1 represents the absolute values of frequency shifts measured for the different metallophthalocyanines coated on quartz crystals towards $500 \mathrm{ppm}$ of toluene at room temperature.

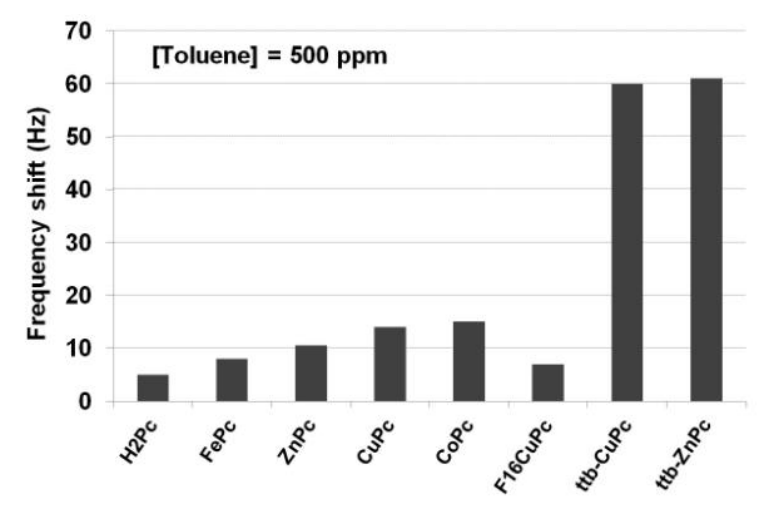

Fig. 1. Responses of different phthalocyaninesbased QCM sensors to 500 ppm of toluene at room temperature.

Despite the high delocalization of $\pi$-electrons on phthalocyanine macrocycles, the sensitivity of the unsubstituted phthalocyanine-based sensors remains low, not higher than $15 \mathrm{~Hz}$. Moreover, response and recovery times are higher than 10 minutes in these experimental conditions. Similar results were obtained towards toluene at the same concentration 
except higher sensitivities, from 25 to $40 \mathrm{~Hz}$. Experimental results highlight that the influence of the central metal atom included into phthalocyanine macrocycle on sensing characteristics is not predominant.

In contrast, the substitution of peripheral hydrogen atoms of phthalocyanine macrocycle by electroactive groups can strongly modulate the sensitivity. In fig.1, the intercomparison between unsubstituted $\mathrm{CuPc}, \mathrm{F}_{16} \mathrm{CuPc}$ and ttb$\mathrm{CuPc}$ clearly established the enhancement provided by electrodonor groups. While the substitution of all the peripheral hydrogen atoms of $\mathrm{CuPc}$ by electronegative ones (fluorine) decreases by two the sensor response, the substitution by electrodonor groups like tert-butyl ligands leads to an increase by four in the sensitivity. Similar conclusions were established with zinc phthalocyanine as depicted in fig.2. Moreover, improvements of response and recovery times are also noticeable, those ones decreasing to 3 minutes. All experimental results show that the electrodonor tert-butyl groups lead to strengthened $\pi$-stacking interactions, these remaining sufficiently weak to enable an easy and fast desorption of gaseous molecules even at room temperature.

In the field of chemical sensors implementing sensitive materials, the structure and the morphology of thin films are crucial characteristics on sensor behavior. Thus, to correlate sensing properties to thin films features, layered tetra-tert butyl and unsubstituted copper phthalocyanines were characterized by scanning electron microscopy. SEM micrographs are reported in Fig.2. The incidence of tert-butyl groups on the morphology of thin films is manifest. If unsubstituted CuPc layers exhibits at the macroscopic scale a columnar structure with local $\beta$-phase arrangement, ttb-CuPc forms larger molecular islands. Moreover, complementary X-Ray diffraction analysis has revealed that aggregates were highly amorphous. The tert-butyl ligands disturb the of staking of phthalocyanine macrocycles, are responsible of the amorphous character of thin layers and consequently lead to more active sites available for gas adsorption. This statement is supported by sensitivity measurements versus layer thickness. Indeed, for CuPc layers from $100 \mathrm{~nm}$ to $400 \mathrm{~nm}$ of thickness, frequency variations of coated QCM depend from BTX concentration but not from layer thickness. For ttb-CuPc sensing devices, response increases linearly with layer thickness. Gas adsorption on many active sites distributed into all the volume is certified.
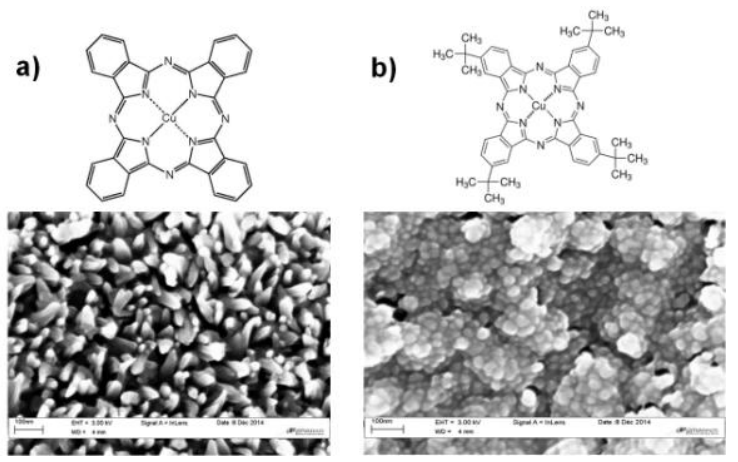

Fig. 2. SEM micrographs of a) unsubstituted copper phthalocyanine and b) tetra-tert-butyl copper phthalocyanine thin films

Grafted on phthalocyanine macrocycles, we have pointed out that tert-butyl groups strongly enhance the sensitivity of these molecular materials towards BTX. Another key specification of gas sensor being the selectivity, calibration of ttb-CuPc-based QCM sensors towards target analytes and interfering gases were made. No significant response was measured with $\mathrm{CO}, \mathrm{NO}_{2}$ or $\mathrm{H}_{2} \mathrm{~S}$. Calibrations were then performed to each target gases to assess the discrimination level reached. The comparison between sensor responses for different concentration of benzene, toluene and xylene in the $30-500$ ppm range is depicted in Fig.3. At first, a linear dependence between frequency shift of coated quartz and BTX concentration can be underlined. Secondly, threshold of detection as well as resolution are better than $30 \mathrm{ppm}$. Complementary experiments in the 1-30 ppm range showed that these are close to $1 \mathrm{ppm}$. Thirdly, we benefit from a high level of repeatability, consecutive exposures to the same concentration giving identical responses. Nevertheless, although calibration curves are separate, discriminated measurements of benzene, toluene and xylenes remains not likely. Innovative selective filters or pre-concentrators upstream the sensor could be attractive approaches to overcome this drawback.

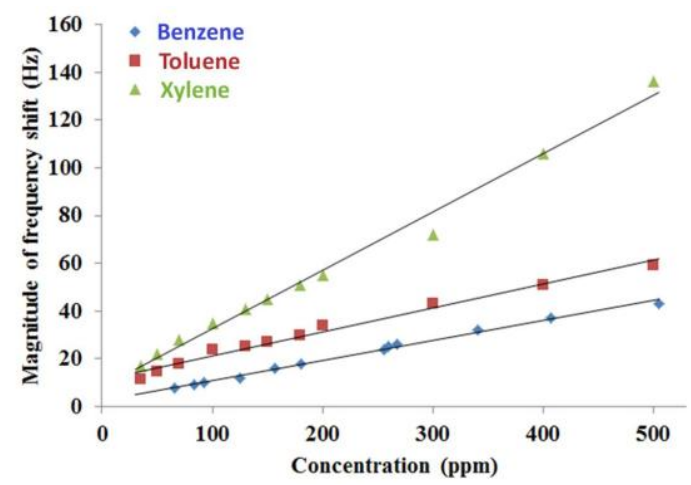

Fig. 3. Responses towards benzene, toluene and xylene of ttb-CuPc-based QCM sensor at room temperature in the 35-500ppm concentration range. 
These strategies will be investigated in the context of an exploratory project, granted by the CNRS, on the selective analysis of monoaromatic hydrocarbons levels in atmosphere (ASTHMAA project - PEPS FaiDoRA 2015). The exploration fields are twofold: i) implementation of functionalized and mesoporous nanocarbonaceous matrix as selective filtering materials and ii) development of selective thermodesorption matrix for the sequential measurement of BTEX gases. Conclusions of these investigations will be given as soon as possible.

\section{Conclusion}

Experimental results obtained on QCM sensors coated with metallophthalocyanines towards benzene, toluene and xylene vapors highlight:

- no significant effect of the central metal atom in phthalocyanine macrocycle on the sensing properties,

- the predominant role of electrodonor groups on thin film morphology and sensor responses,

- the 6-10 times higher sensitivity for tetra-tertbutyl metallophthalocyanines as compared to unsubstituted ones and faster kinetics of responses,

- response and recovery times close to 3 minutes at room temperature,

- the low cross-sensitivities for $\mathrm{CO}, \mathrm{NO}_{2}$ and $\mathrm{H}_{2} \mathrm{~S}$ and the partial selectivity achieved to BTX.

The discriminated measurement of each monoaromatic hydrocarbon remains the challenge to overcome. The development of specific chemical filters or selective preconcentrators implemented upstream the sensing devices could be innovative solutions to reach selective detection. This is the ambitious objective of the ASTHMAA exploratory project granted by the CNRS we coordinate.

\section{Acknowledgements}

A. Kumar thanks the Ministry of Higher Education, France to provide doctoral fellowship. Authors would like to address their acknowledgements to the National Centre for Scientific Research (CNRS) for their financial support on the exploratory project named ASTHMAA (PEPS FaiDoRA 2015).

\section{References}

[1] K. Straif, A. Cohen, J. Samet, Air Pollution and Cancer, IARC Scientific Publication 161 (2013); ISSN: 0300-5085

[2] D. Loomis et al., The carcinogenicity of outdoor air pollution, The Lancet Oncology 14, 1262-1263 (2013); doi: 10.1016/S1470-2045(13)70487-X
[3] G. A. Pilidis, S. P. Karakitsios, P. A

Kassomenos, BTX measurements in a mediumsized European city, Atmospheric Environment 39, 6051-6065 (2005); doi:

10.1016/j.atmosenv.2005.06.044

[4] C.R. Martinez, B.L. Iverson, Rethinking the term "pi-stacking", Chemical Science 3, 2191-2201 (2012); doi: 10.1039/C2SC20045G.

[5] C.A. Hunter, K.R. Lawson, J. Perkins, C.J. Urch, Aromatic interactions, Journal of the Chemical Society, Perkin Transactions 2, 651-669 (2001); doi: 10.1039/B008495F

[6] A. Kumar et al., Tetra-tert-butyl copper phthalocyanine-based QCM sensor for toluene detection in air at room temperature, Sensors and Actuators B 210, 398-407 (2015); doi: 10.1016/j.snb.2015.01.010 